\title{
Long-Term Outcomes of Induction Therapies with Alemtu- zumab, Basiliximab or Methylprednisolone in Kidney Trans- plant Patients with Delayed Graft Function
}

\section{Allen Zhang ${ }^{1}$, Beatrice Zhang' ${ }^{2}$ Yaozhong Liu ${ }^{3}$, Mary Killackey ${ }^{1}$, Anil Paramesh ${ }^{1}$, Joseph Buell', Brent Alper', Fred Teran', Adrian Baudy' ${ }^{1}$ and Rubin Zhang ${ }^{1 *}$}

${ }^{1}$ Tulane University School of Medicine, New Orleans, LA, USA

${ }^{2}$ University of Pennsylvania School of Arts and Sciences, Philadelphia, PA, USA

${ }^{3}$ Tulane University School of Public Health and Tropic Medicine, New Orleans, LA, USA

\begin{abstract}
Introduction: Delayed graft function (DGF) after kidney transplant is associated with high risk of acute rejection (AR) and graft failure. The optimal immunosuppressive strategy for patients with DGF remains unknown.

Material and method: We compare the 5-year outcomes of induction therapies with methylprednisolone $(\mathrm{n}=58)$, basiliximab $(\mathrm{n}=56)$ or alemtuzumab $(\mathrm{n}=98)$ in patients with DGF. Maintenance was tacrolimus and mycophenolate with prednisone in methylprednisolone and basiliximab groups or without prednisone in alemtuzumab group. Protocol biopsies were performed in all patients.

Results: 5-year biopsy-confirmed AR rates were significantly different among the 3 groups $(39.7 \%, 28.6 \%$ and $20.4 \%$ in methylprednisolone, basiliximab and alemtuzumab group, respectively; $\mathrm{p}=0.034$ ). There was a trend of difference in Kaplan-Meier estimated 5-year graft survivals among the 3 groups $(65.5 \%, 71.4 \%$ and $80.6 \%$, respectively; log rank $\mathrm{p}=0.07)$. Alemtuzumab group had a lowest incidence of AR and highest graft survival. The 5-year patient survivals were not statistically different in the 3 groups $(75.9 \%, 82.1 \%$ and $84.7 \%, \log$ rank $p=0.4)$. Multivariable analysis using methylprednisolone induction as control indicated that alemtuzumab (HR 0.36, 95\% CI 0.13-0.85; p $=0.036)$ and basiliximab (HR 0.67, 95\% CI 0.20-0.97; p = 0.023) were associated with lower risk of AR.
\end{abstract}

Conclusion: Alemtuzumab induction decreases AR rate in kidney transplant patients with DGF and can improve longterm graft survival in comparison to other induction therapies.

Keywords

Induction therapy, Delayed graft function, Kidney transplant, Acute rejection, Graft survival, Basiliximab, Alemtuzumab, Steroid withdrawal

\section{Introduction}

Delayed graft function (DGF) after kidney transplant is usually defined as the need of dialysis support in the first post-operative week [1-3]. DGF is predominantly caused by ischemia-reperfusion injury (IRI). The severity of IRI varies, which affects the quality and function of allograft $[1,2]$. DGF not only prolongs the hospital stay, increases the financial cost of care, it is also associated with poor graft function, inferior graft survival and patient survival [1-6].

Interestingly, DGF also increases the risk of acute rejection (AR) [7-9]. The development of AR in the set-

*Corresponding author: Rubin Zhang, MD, FASN, Section of Nephrology and Hypertension, Department of Medicine, Tulane University School of Medicine, 1430 Tulane Ave, New Orleans, LA 70112, USA, Tel: 504-988-1457, Fax: 504988-1105

Accepted: October 24, 2018; Published online: October 26, 2018

Citation: Zhang A, Zhang B, Liu Y, et al. (2018) LongTerm Outcomes of Induction Therapies with Alemtuzumab, Basiliximab or Methylprednisolone in Kidney Transplant Patients with Delayed Graft Function. J Transplant Surg 1(1):26-32

Copyright: (c) 2018 Zhang A, et al. This is an open-access article distributed under the terms of the Creative Commons Attribution License, which permits unrestricted use, distribution, and reproduction in any medium, provided the original author and source are credited. 
Citation: Zhang A, Zhang B, Liu Y, et al. (2018) Long-Term Outcomes of Induction Therapies with Alemtuzumab, Basiliximab or Methylprednisolone in Kidney Transplant Patients with Delayed Graft Function. J Transplant Surg $1(1): 26-32$

ting of DGF is usually "silent", as the patients remain dialysis-dependent. It can only be diagnosed by serial protocol biopsies. Without timely recognition and appropriate treatment, AR would contribute to the poor outcome associated with DGF $[5,7,10]$. Successful prevention and/or treatment of AR can decrease the risk of graft loss. However, the ideal immunosuppressive regimen for patients with DGF has not been established and the selection of the optimal induction agent remains speculative [11-19]. Current induction agents include methylprednisolone, interleukin-2 receptor (IR2R) antibody (basiliximab) and lymphocyte-depleting antibody (antithymocyte globulin and alemtuzumab). Previously, we reported a 1-year comparison study of three different induction agents, methylprednisolone, basiliximab or alemtuzumab in patients with DGF [20]. We found that alemtuzumab induction was associated with the lowest risk of AR and probably a better graft survival. The aim of this study is to compare the long-term (5-year) results of the three induction therapies in kidney transplant patients with DGF.

\section{Patients and Methods}

\section{Study population}

As stated in our previous report, patients were identified using the transplant center database at Tulane University Hospital \& Clinic [20]. From 2006 to 2013, there were consecutive 573 adult patients who underwent a primary kidney transplant from deceased donors. Among them, 212 (37\%) had DGF and were included in the study. These patients were divided into three groups according to their induction therapies. There were 58 patients in methylprednisolone (steroid) group, 56 patients in the basiliximab group, and 98 patients in the alemtuzumab group. Those excluded from this study were also described in our previous report [20]. Our protocol for patients with DGF included a kidney biopsy before patients were discharged home after the transplant surgery. Serial protocol biopsies were subsequently performed every 2 to 4 weeks until either graft recovered function and dialysis was discontinued or graft was deemed primary failure after 3 months of transplant. Kidney biopsy was typically performed with the real-time ultrasound guidance. Bard monopty disposable core biopsy instrument kit with the needle size of $18 \mathrm{~g}$ $\times 20 \mathrm{~cm}$ was used under local anesthetic.

\section{Immunosuppressive therapy}

From 2006 to 2010, methylprednisolone or basiliximab was used as induction agent and all patients received the traditional triple-maintenance of tacrolimus, mycophenolate and prednisone. In 2011, our program changed the immunosuppressive protocol, and alemtuzumab was used as induction antibody in order to adopt a contemporary early steroid-withdrawal. The details of our immunosuppressive protocols, diagnosis and treatment of AR as well as infection prophylaxes were described in the previous report [20].

Table 1: Demographic characteristics of transplant patients between steroid, basiliximab and alemtuzumab inductions.

\begin{tabular}{|c|c|c|c|}
\hline & Steroid $(n=58)$ & Basiliximab $(n=56)$ & Alemtuzumab $(n=98)$ \\
\hline Age mean $\pm \mathrm{sd}(\mathrm{y})$ & $48.2 \pm 14.2$ & $46.8 \pm 15.4$ & $47.3 \pm 14.4$ \\
\hline \multicolumn{4}{|l|}{ Gender (\%) } \\
\hline male & 57 & 54 & 55 \\
\hline female & 43 & 46 & 45 \\
\hline \multicolumn{4}{|l|}{ Race (\%) } \\
\hline black & 60 & 64 & 62 \\
\hline non-black & 40 & 36 & 38 \\
\hline BMI $\left(\mathrm{kg} / \mathrm{m}^{2}\right)$ & $27.5 \pm 6.6$ & $28.2 \pm 6.1$ & $27.8 \pm 5.9$ \\
\hline Peak PRA (\%) & $13.2 \pm 24.6$ & $16.8 \pm 27.3$ & $14.9 \pm 23.7$ \\
\hline HLA mismatch & $3.6 \pm 1.5$ & $4.3 \pm 1.6$ & $4.1 \pm 1.4$ \\
\hline \multicolumn{4}{|c|}{ Causes of ESRD (\%) } \\
\hline diabetes & 26 & 30 & 28 \\
\hline hypertension & 40 & 34 & 37 \\
\hline nephritis & 17 & 14 & 14 \\
\hline PCKD & 7 & 9 & 8 \\
\hline others & 10 & 13 & 13 \\
\hline CIT (hrs) & $16.3 \pm 7.6$ & $18.9 \pm 6.9$ & $17.7 \pm 6.3$ \\
\hline ECD (\%) & 31 & 39.3 & 34.7 \\
\hline
\end{tabular}

Significant $p$-values:

Peak PRA: $p=0.01$ for steroid group vs. basiliximab group; $p=0.03$ for basiliximab group vs. alumtuzmab group; CIT: $p=0.04$ for steroid group vs. basiliximab group; $p=0.02$ for basiliximab group vs. alumtuzmab group.

Non-significant $p$-values:

Age: $p=0.25$; Gender: $p=0.47 ;$ Race: $p=0.81 ;$ BMI: $p=0.52$; Cause of ESRD: $p=0.95 ;$ ECD: $p=0.49$. 
Citation: Zhang A, Zhang B, Liu Y, et al. (2018) Long-Term Outcomes of Induction Therapies with Alemtuzumab, Basiliximab or Methylprednisolone in Kidney Transplant Patients with Delayed Graft Function. J Transplant Surg $1(1): 26-32$

\section{Statistical analysis}

The outcome measures included: 1) 5-year cumulative incidence of biopsy-confirmed and clinically-treated AR; 2) 5-year kidney graft and patient survival; 3) Independent risk factors of AR. Statistical analyses were performed using SAS version 9.3 software (SAS Institute Inc, Cary, NC, USA). Chi-squared or Fisher exact test was used for count data, t-test was used for continuous measures. Product-limit estimates of survival curves were generated by the Kaplan-Meier method and the survival difference was analyzed by log-rank test. Multivariable logistic regression analysis with a stepwise variable selection was used for examining risk factors of AR. A p-value $<0.05$ was considered statistically significant.

\section{Results}

Table 1 summarizes the patient demographic characteristics at the time of transplant. There was no significant difference between the 3 groups in terms of patient age, gender, race, body mass index (BMI), the causes of ESRD and ECD. However, the basiliximab group had a higher peak PRA and longer CIT when compared with steroid group or alemtuzumab group.

There were significant differences in the incidences of biopsy-confirmed and clinically-treated AR among the
3 groups, and the 5-year cumulative incidences of AR were $39.7 \%, 28.6 \%$ and $20.4 \%$ in steroid, basiliximab and alemtuzumab group respectively (Figure $1 ; \log \operatorname{rank} p=$ $0.034)$. When comparison was performed between each 2 groups, statistical significance was detected between steroid and alemtuzumab groups $(39.7 \%$ vs. $20.4 \%, \mathrm{p}=$ 0.015). The incidence of AR also trended (but not statistically) higher in steroid group than basiliximab group ( $39.7 \%$ vs. $28.6 \%, p=0.34)$, and in basiliximab group than alemtuzumab group ( $28.6 \%$ vs. $20.4 \%, p=0.29)$. The types of AR in each group were summarized in Table 2.

The Kaplan-Meier estimated 5-year graft survival rates were $65.5 \%, 71.4 \%$ and $80.6 \%$ in the steroid, basiliximab and alemtuzumab groups, respectively. Despite of the obvious trend, the differences did not reach a statistical significance (Figure 2, log-rank $p=0.07$ ). Alemtuzumab group had a numerically superior graft survival compared to steroid group ( $80.6 \%$ vs. $65.5 \%, \mathrm{p}=0.06$ ). The causes of graft loss in each group are listed in Table 2. The Kaplan-Meier estimated 5-year patient survivals in the 3 groups were not significantly different (Figure 3 , log-rank $\mathrm{p}=0.4$ ). The estimated 5 -year patient survival rates were $75.9 \%, 82.1 \%$ and $84.7 \%$ in the steroid, basiliximab and alemtuzumab groups, respectively. The causes of patient death in each group are summarized in Table 2.

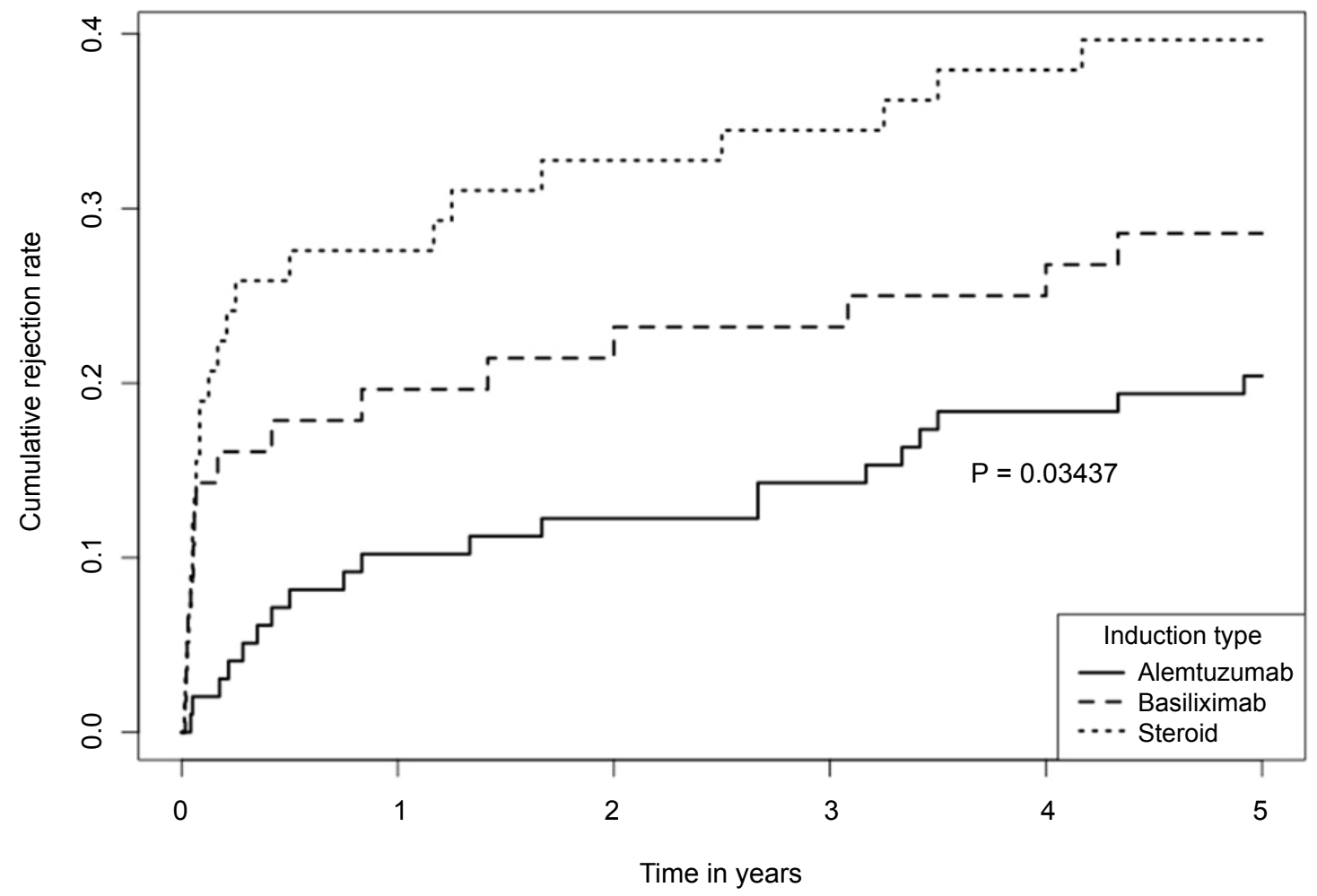

Figure 1: The 5-year cumulative incidences of biopsy-confirmed and clinically-treated acute rejection. The rejection rates were $39.7 \%, 28.6 \%$ and $20.4 \%$ in steroid, basiliximab and alemtuzumab groups respectively. The rejection rate was significantly higher in steroid group than in alemtuzumab group $(p=0.015)$. It was trended higher in steroid group compared with basiliximab group $(p=0.34)$, and in basiliximab group compared with alemtuzumab group $(p=0.29)$. 
Citation: Zhang A, Zhang B, Liu Y, et al. (2018) Long-Term Outcomes of Induction Therapies with Alemtuzumab, Basiliximab or Methylprednisolone in Kidney Transplant Patients with Delayed Graft Function. J Transplant Surg $1(1): 26-32$

Table 2: Summary of 5-year rejection types, causes of graft loss and patient death.

\begin{tabular}{|c|c|c|c|}
\hline & Steroid $(n=58)$ & Basiliximab $(n=56)$ & Alemtuzumab $(n=98)$ \\
\hline Acute Rejection, n (\%) & $24(39.7)$ & $16(28.6)$ & $20(20.4)$ \\
\hline ACR & 12 & 7 & 7 \\
\hline AMR & 4 & 3 & 5 \\
\hline$A C R+A M R$ & 8 & 6 & 8 \\
\hline Total Graft Loss, n (\%) & $20(34.5)$ & $16(28.6)$ & $19(19.4)$ \\
\hline \multicolumn{4}{|l|}{ Causes of Graft Loss: } \\
\hline DWFG & 9 & 7 & 8 \\
\hline Rejection & 5 & 5 & 4 \\
\hline CAN & 4 & 3 & 4 \\
\hline Infection & 2 & 1 & 3 \\
\hline Total Patient Death, n (\%) & $14(24.1)$ & $10(17.9)$ & $15(15.3)$ \\
\hline \multicolumn{4}{|l|}{ Cause of Death } \\
\hline CVD & 10 & 8 & 9 \\
\hline Infection & 3 & 2 & 4 \\
\hline Cancer & 1 & 0 & 1 \\
\hline Others & 0 & 0 & 1 \\
\hline
\end{tabular}

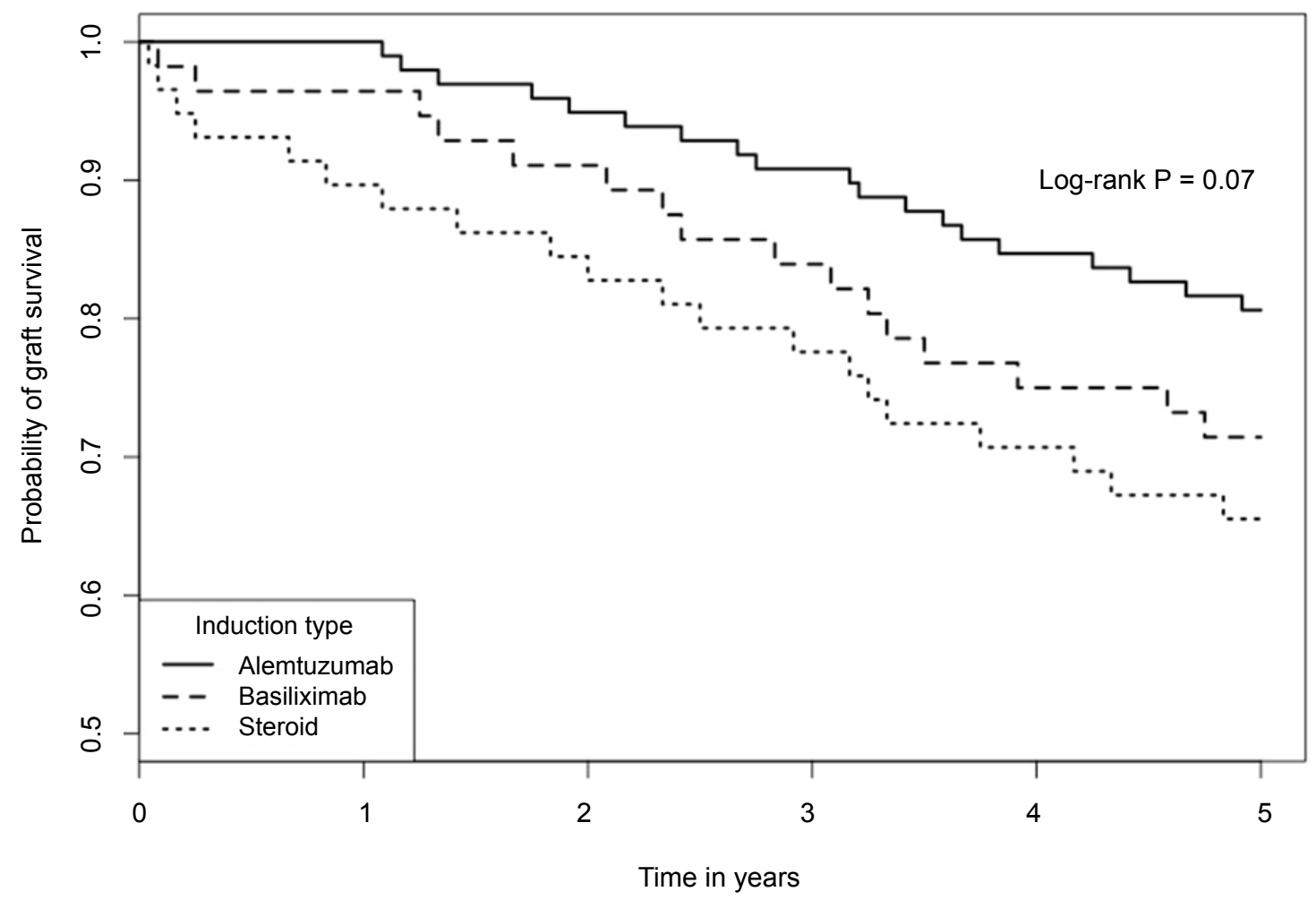

Figure 2: Kaplan-Meier analysis of 5 -year graft survival. The estimated graft survival was $65.5 \%, 71.4 \%$ and $80.6 \%$ in steroid, basiliximab and alemtuzumab groups respectively. Steroid group had a numerically lower graft survival than basiliximab group $(p=0.451)$ and alemtuzumab group $(p=0.068)$, but these differences did not reach statistical significance.

Table 3: Multivariable analysis of risk factors for acute rejection.

\begin{tabular}{|l|l|l|l|}
\hline & Hazard ratio & $\mathbf{9 5 \%} \mathbf{~ C l}$ & $\boldsymbol{p}$-value \\
\hline Race (black vs. non-black) & 3.02 & $1.08-9.30$ & $<0.001$ \\
\hline Basiliximab (vs. steroid) & 0.67 & $0.20-0.97$ & 0.023 \\
\hline Alemtuzumab (vs. steroid) & 0.36 & $0.13-0.85$ & 0.036 \\
\hline
\end{tabular}

The risk factors for AR in patients with DGF were We modeled AR as dependent variable and all other facexamined by multivariable logistic regression analyses. tors (including age, gender, ethnicity, peak PRA, HLA 
Citation: Zhang A, Zhang B, Liu Y, et al. (2018) Long-Term Outcomes of Induction Therapies with Alemtuzumab, Basiliximab or Methylprednisolone in Kidney Transplant Patients with Delayed Graft Function. J Transplant Surg $1(1): 26-32$

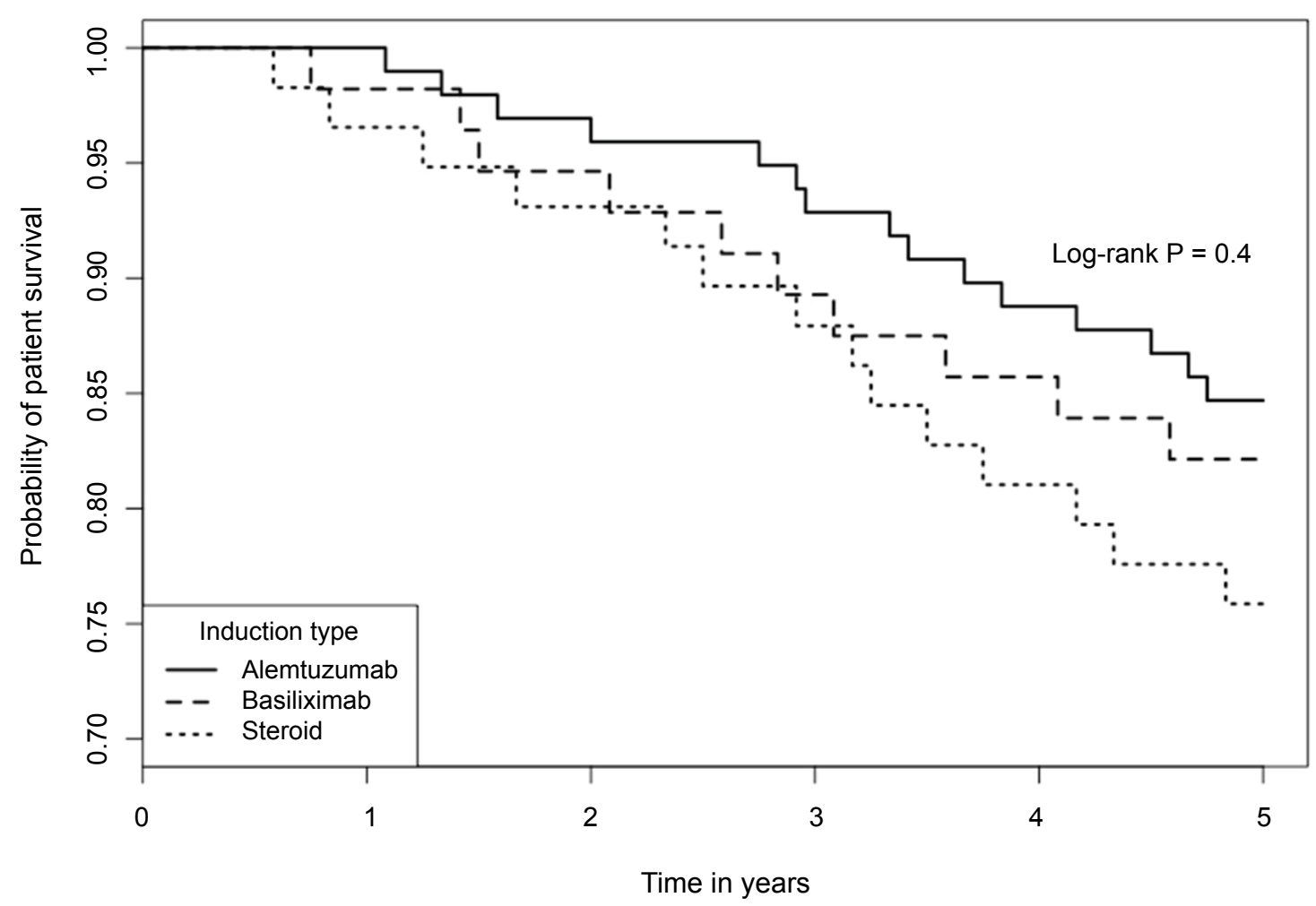

Figure 3: Kaplan-Meier analysis of 5-year patient survival. The estimated patient survival was $75.9 \%, 82.1 \%$ and $84.7 \%$ in steroid, basiliximab and alemtuzumab groups respectively. The patent survival difference among the 3 groups was not statistically significant (log-rank $p=0.4$ ).

mismatch, CIT, ECD, BMI, causes of ESRD and types of induction) as independent variables. A stepwise variable selection method was used for testing risk factors of AR. The identified significant factors are listed in Table 3. Not surprisingly, black ethnicity was found as a significant risk of AR (HR 3.02, 95\% Cl 1.08-9.30; p < 0.001). When steroid induction was used as control, we found that basiliximab induction was associated with lower risk of AR (HR 0.67, 95\% Cl 0.20-0.97; p = 0.023), and this was also true for alemtuzumab induction (HR $0.36,95 \%$ $\mathrm{Cl} 0.13-0.85 ; \mathrm{p}=0.036$ ).

\section{Discussion}

The incidence of DGF is rising as transplant programs increasingly use kidneys from ECD and donation after circulatory death [1-5]. The deceased donor kidney is subjected to injury at every step along the path from donor death, prolonged ischemic time to reperfusion injury [1-3]. IRI is an acute non-specific inflammatory process. This innate immune response can however, activate an adaptive immune response by up-regulating the expression of adhesion molecules, cytokines and HLA. It can also increase antigen presentation to T-cells, therefore, promote allo-sensitization and increase the risk of $A R$ [1,2,21-23]. Qureshi F, et al. reported that AR was very common in kidneys with DGF (57.2\%) compared with kidneys without DGF (15.1\%). The risk of graft loss from AR was 2.91 (HR, 95\% CI 1.60-5.27, $\mathrm{p}=0.0004)$, and much of the reduced graft survival could be explained by the development of silent AR, which could only be detected by protocol biopsy [7]. Arias $\mathrm{M}$ also reported high incidence of AR in patients with DGF (47\%), presensitization increased the risk of DGF and both together led to the worst graft survivals [24]. High incidence of AR in patients with DGF contributes to their poor outcomes including graft dysfunction, graft loss as well as patient death $[4,6,8,9]$.

The optimal immunosuppressive protocol for patients with DGF remains speculative. Potent antibody induction theoretically could be beneficial for patients with DGF by suppressing both the innate and adaptive immune responses. The effect of introoperative versus postoperative administration of thymoglobulin induction was studied in deceased donor kidney transplants. Intraoperative thymoglobulin was associated with significantly lower incidence of DGF $(14.8 \%$ vs. $35.5 \%$, p $=0.05)$, better graft function and shorter hospitalization [13]. In a randomized control trial, Brennan DC, et al. compared thymoglobulin versus basiliximab induction in deceased donor kidney transplants at high risk for DGF and AR. They found no significant difference in the incidence of DGF ( $40.4 \%$ vs. $44.5 \%, \mathrm{p}=0.54$ ) between the two induction groups [16].

In the previous short-term study, we reported the 1-year outcome of 3 different induction therapies in 
Citation: Zhang A, Zhang B, Liu Y, et al. (2018) Long-Term Outcomes of Induction Therapies with Alemtuzumab, Basiliximab or Methylprednisolone in Kidney Transplant Patients with Delayed Graft Function. J Transplant Surg $1(1): 26-32$

patients with established DGF. Compared with steroid induction, antibody induction with either basiliximab or alemtuzumab was associated with significantly lower incidence of AR [20]. In this follow-up study, we found that the 5-year cumulative incidences of AR remained significantly different among the 3 groups (log rank $\mathrm{p}$ $=0.034)$, especially between steroid and alemtuzumab groups $(39.7 \%$ vs. $20.4 \%, p=0.015)$. There was an obvious trend of better long-term graft survival in alemtuzumab group compared to other two groups, but the difference did not reach statistical significance likely due to small sample sizes $(\log$-rank $p=0.07)$. Despite of this limitation, alemtuzumab group had a numerically superior 5-year graft survival compared to steroid group $(80.6 \%$ vs. $65.5 \%, p=0.06)$. When the risk factors for AR were examined by multivariable logistic regression analyses, black ethnicity was found to be associated with significantly higher risk of AR. When steroid induction was used as control, we found that antibody induction with either alemtuzumab (HR 0.36, 95\% Cl 0.13-0.85; p $=0.036)$ or basiliximab induction ( $\mathrm{HR} 0.67,95 \% \mathrm{Cl} 0.20$ $0.97 ; \mathrm{p}=0.023$ ) was associated with lower risk of AR.

In literature, Goncalves LF, et al. reported a shortterm study of 148 Brazilians with DGF: 90 of them received basiliximab induction and remaining 58 patients received steroid induction as the control group [14]. Both groups were maintained with triple combination of steroid, CNI and MMF. Compared with the steroid control, basiliximab induction demonstrated a lower incidence of steroid-resistant AR $(20.9 \%$ vs. $6 \%$, p $=$ $0.017)$ and AMR ( $7 \%$ vs. $0 \%, \mathrm{p}=0.038)$ at 1 year. The 1-year graft survival was significantly higher in basiliximab group than the control group ( $92.8 \%$ vs. $80.4 \%$, p $=0.028$ ) [14]. To our knowledge, this was the only study published by others to compare induction therapies in patients with DGF. Our current report is the first longterm study and our patients are predominantly African Americans (more than 60\% in each group) and are at higher risk of AR. More importantly, our study includes a group of alemtuzumab induction with a contemporary protocol of steroid-withdrawal maintenance. Alemtuzumab is a humanized anti-CD52 monoclonal antibody and it produces a profound depletion of both $\mathrm{T}$ - and B- lymphocytes. Previous studies suggested that alemtuzumab induction could permit patients to be maintained on unconventional strategies such as steroid-free $[25,26]$ or steroid-withdrawal [27] or low-dose of CNI [26]. The recent Cochrane review also concluded that alemtuzumab was associated with lower risk of AR compared to antithymocyte globulin in the contest of steroid minimization protocols [19]. However, its benefit on patients with DGF has not been reported previously. The initial data from the 3C study suggested no significant difference of the incidences of DGF between alemtuzumab induction and basiliximab induction ( $30 \%$ vs. $24 \%$; OR $1.35,95 \%$ CI 1.00-1.83; $\mathrm{p}=0.054)$. But alemtuzumab induction did reduce the primary outcome of biopsy-proven AR [26]. Both of our short and long-term studies are the first ones demonstrating a protective benefit of alemtuzumab induction in patients with established DGF. In addition, our patients in the alemtuzumab group were maintained on tacrolimus and MMF without steroid, while patients in other two groups received traditional triple-maintenance. Therefore, our data also indicate that with alemtuzumab induction, the contemporary steroid-withdrawal protocol can be successfully used in patients with DGF. Our study is limited by its single center data, relative small sample size and retrospective nature. As the diagnosis of DGF is retrospective in nature, it would be impossible to conduct a prospective and controlled comparison of various induction therapies in DGF. To our knowledge, there is no such prospective study published in literature.

\section{Conclusion}

Our long-term study supports the usage of potent antibody induction with alemtuzumab in patients with DGF, as it significant decreases the incidence of AR and may provide a long-term graft survival benefit. Also, alemtuzumab induction can allow patients with DGF to be successfully maintained with contemporary steroid-withdrawal protocol.

\section{Conflict of Interest}

The authors declare that they have no conflict of interest.

\section{Statement of Human Rights}

This study was approved by the Institutional Review Board (IRB) of Tulane University.

\section{Informed Consent}

Informed consent from individual participant is not indicated as it is a retrospective analysis and there is no identifying information about any participant in the article.

\section{References}

1. Siedlecki A, Irish W, Brennan D (2011) Delayed graft function in the kidney transplant. Am J Transplant 11: 2279-2296.

2. Sharif A, Richard B (2013) Delayed graft function after kidney transplantation: The clinical perspective. Am J Kidney Dis 62: 150-158.

3. Mallon D, Summers D, Bradley A, et al. (2013) Defining delayed draft function after renal transplantation: Simplest is best. Transplantation 96: $885-889$.

4. Boom H, Mallat M, Fijter J, et al. (2000) Delayed graft function influences renal function, but not survival. Kidney Int 58: 859-866. 
Citation: Zhang A, Zhang B, Liu Y, et al. (2018) Long-Term Outcomes of Induction Therapies with Alemtuzumab, Basiliximab or Methylprednisolone in Kidney Transplant Patients with Delayed Graft Function. J Transplant Surg $1(1): 26-32$

5. Nagaraja P, Roberts G, Stephens M, et al. (2012) Influence of delayed graft function and acute rejection on outcomes after kidney transplantation from donors after cardiac death. Transplantation 94: 1218-1223.

6. Tapiawala SN, Tinckam KJ, Cardella CJ, et al. (2010) Delayed graft function and the risk for death with a functioning graft. J Am Soc Nephrol 21: 153-161.

7. Qureshi F, Rabb H, Kasiske B (2002) Silent acute rejection during prolonged delayed graft function reduces kidney allograft survival. Transplantation 74: 1400-1404.

8. Yarlagadda SG, Coca SG, Formica RN Jr, et al. (2009) Association between delayed graft function and allograft and patient survival: A systematic review and meta-analysis. Nephrol Dial Transplant 24: 1039-1047.

9. Ojo AO, Wolfe RA, Held PJ, et al. (1997) Delayed graft function: Risk factors and implications for renal allograft survival. Transplantation 63: 968-974.

10. Troppmann C, Gillingham KJ, Gruessner RW, et al. (1996) Delayed graft function in the absence of rejection has no long-term impact. Transplantation 61: 1331-1337.

11. Boletis J, Balitsari A, Filiopoulos V, et al. (2005) Delayed renal graft function: the influence of immunosuppression. Transplantation Proceedings 37: 2054-2059.

12. Ponticelli C (2014) Ischaemia-reperfusion injury: A major protagonist in kidney transplantation. Nephr Dia Transplant 29: 1134-1140.

13. Goggins WC, Pascual MA, Powelson JA, et al. (2003) A prospective randomized clinical trial of intraoperative versus postoperative thymoglobulin in adult deceased renal transplant recipients. Transplantation 76: 798-802.

14. Goncalves L, Ribeiro A, Berdichevski R, et al. (2007) Basiliximab improves graft survival in renal transplant recipients with delayed graft function. Transplant Proc 39: 437-438.

15. Chouhan K, Zhang R (2012) Antibody induction therapy in adult kidney transplantation, a controversy continues. World J Transplant 2: 19-26.

16. Brennan DC, Daller JA, Lake KD, et al. (2006) Rabbit antithymocyte globulin versus basiliximab in renal transplantation. N Engl J Med 355: 1967-1977.
17. Webster AC, Ruster LP, McGee RG, et al. (2010) Interleukin 2 receptor antagonists for kidney transplant recipients. Cochrane Database Syst Rev, 1.

18. Noel C, Abramowicz D, Durand D, et al. (2009) Daclizumab versus antithymocyte globulin in high-immunological-risk renal transplant recipients. J Am Soc Nephrol 20: 13851392.

19. Hill P, Cross NB, Barnett ANR, et al. (2017) Polyclonal and monoclonal antibodies for induction therapy in kidney transplant recipients. Cochrane Database of Systematic Reviews 1 .

20. Umber A, Killackey M, Paramesh A, et al. (2017) A comparison of three induction therapies on patients with delayed graft function after kidney transplantation. J Nephrol 30: 289-295.

21. Kumbala D, Zhang R (2013) The essential concept of transplant immunology for clinical practice. World J Transplant 3: 113-118.

22. Zhang $R$ (2018) Donor specific antibodies in kidney transplant recipients. Clin J Am Soc Nephrol 13: 182-192.

23. Peeters $P$, Vanholder $R$ (2008) Therapeutic interventions favorably influencing delayed and slow graft function in kidney transplantation: mission impossible? Transplantation 85: 31-37.

24. Arias M (2003) Impact of the delayed graft function in hypersensitized kidney transplant patients. Transplant Proc 35: 1655-1657.

25. Kaufman DB, Leventhal JR, Axelrod D, et al. (2005) Alemtuzumab induction and prednisone-free maintenance immunotherapy in kidney transplantation: comparison with basiliximab induction--long-term results. Am J Transplant 5: 2539-2548.

26. Haynes R, Harden P, Judge P, et al. (2014) Alemtuzumab-based induction treatment versus basiliximab-based induction treatment in kidney transplantation (the $3 \mathrm{C}$ Study): A randomised trial. Lancet 384: 1684-1690.

27. Hanaway MJ, Woodle ES, Mulgaonkar S, et al. (2011) Alemtuzumab induction in renal transplantation. $\mathrm{N}$ Engl $\mathrm{J}$ Med 364: 1909-1919. 